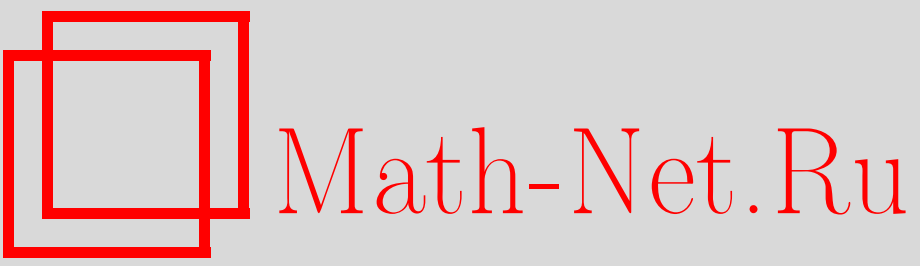

Л. В. Ильичев, Кинетическая модель переброса спиновых корреляций, ТМФ, 2001, том 127, номер 1, 168-176

DOI: https://doi.org/10.4213/tmf454

Использование Общероссийского математического портала Math-Net.Ru подразумевает, что вы прочитали и согласны с пользовательским соглашением

http://www.mathnet.ru/rus/agreement

Параметры загрузки:

IP : 18.207 .199 .55

26 апреля 2023 г., 13:31:01

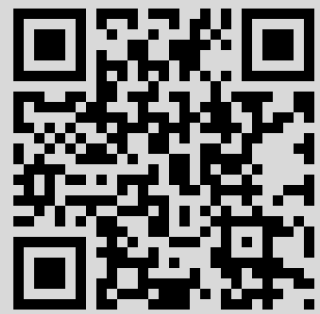




\section{КИНЕТИЧЕСКАЯ МОДЕЛЬ ПЕРЕБРОСА СПИНОВЫХ КОРРЕЛЯЦИЙ}

Развита предложенная ранее кинетическая модель рекомбинации радикальных пар, демонстрирующая явление "переброса" квантовых спиновых корреляций для произвольного источника скоррелированных пар. Обсуждаются проблемы учета информации от несвершившихся актов рекомбинации. Намечен возможный путь развития последовательной модели с перебросом спиновых корреляций.

\section{1. ВВЕДЕНИЕ}

Составная квантовая система $A \cup B$ способна демонстрировать коррелящии между своими подсистемами $A$ и $B^{1)}$. Существует следующая классификация типов корреляций. Прежде всего, возможна тривиальная ситуация полного отсутствия корреляций, если матрища плотности составной системы факторизуется:

$$
\hat{\varrho}_{A \cup B}=\hat{\varrho}_{A} \otimes \hat{\varrho}_{B}
$$

Корреляции появятся, если матрица плотности $\hat{\varrho}_{A \cup B}$ представима в виде выпуклой линейной комбинации факторизованных членов вида (1.1), взятых со своими весами $p_{i}$ :

$$
\hat{\varrho}_{A \cup B}=\sum_{i} p_{i} \hat{\varrho}_{A}^{(i)} \otimes \hat{\varrho}_{B}^{(i)},
$$

где $p_{i}>0, \sum_{i} p_{i}=1$. Система $A \cup B$ в состоянии (1.2) демонстрирует так называемые классические корреляции. Такие состояния не нарушают, в частности, неравенства Белла [1].

Если матрица плотности системы $A \cup B$ не представима в виде (1.2), то состояние составной системы по определению называется зацепленным (entangled state). Такие состояния важны для квантовой теории информации и квантовой коммуникации.

\footnotetext{
1) Под корреляциями мы понимаем статистическую зависимость результатов измерений, проводимых на подсистемах $A$ и $B$.
}

${ }^{*}$ Институт автоматики и электрометрии СО РАН, Новосибирский государственный университет, Новосибирск, Россия. E-mail: shalagin@iae.nsk.su 
Именно в квантовой теории информации в 1993 году был введен термин "переброс зацепленности" (entanglement swapping) [2] (в более общем случае следует говорить о "перебросе корреляций") для явления, о котором пойдет речь в настоящей работе. Однако, насколько известно автору, впервые частный случай явления переброса квантовых корреляций, имеюший место при рекомбинации спиновых радикальных пар, описал еще в 1985 году Броклехурст [3]. Суть этого примера такова. Рассматривалась среда, в которой рож даются и рекомбинируют пары радикалов - частиш со спином $1 / 2$. Рекомбинация предполагалась возможной только по синглетному каналу, т.е. при суммарном нулевом спине радикальной пары. Заметим, что синглетное состояние является в определенном смысле максимально зацепленным. При встрече и рекомбинации фрагментов $\{1,3\}$ из двух разных синглетных пар $\{1,2\}$ и $\{3,4\}$ оставшаяся пара фрагментов $\{2,4\}$ оказьвается в синглетном состоянии (рис. 1 ), что с очевидностью следует из закона сохранения суммарного спина двух пар. Априорное спиновое состояние пары $\{2,4\}$ до рекомбинации пары $\{1,3\}$ было максимально смешанным и, естественно, не содержало никаких корреляций. Таким образом, в результате акта рекомбинации произошел переброс синглетности (и зацепленности) внутри четверки частиц $\{1,2,3,4\}$ с пар $\{1,2\}$, $\{3,4\}$ на новые пары $\{1,3\}$ и $\{2,4\}$. Первая из этих пар, преврашаясь в продукт реакции рекомбинации, выпадает из дальнейшего рассмотрения.
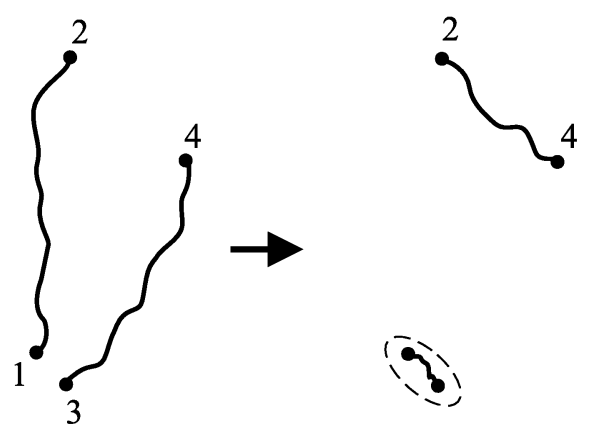

Рис. 1. Переброс зацепленности (синглетности) при перекрестной рекомбинации частиц из двух синглетных пар. Синглетные пары соединены волнистыми линиями. Штриховой линией окружен продукт рекомбинации.

Принципиальным условием, предваряющим какое-либо использование спиновых корреляций, возникающих в состоянии $\hat{\varrho}(2,4)$, является получение сигнала о рекомбинации пары $\{1,3\}$. В литературе это обстоятельство часто трактуется как необходимость наличия двух типов каналов передачи информации при осушествлении переброса зацепленности - классического канала, по которому передается сообщение об исходе эксперимента (в данном случае о свершившемся акте рекомбинации), и так называемых квантовых каналов Эйнштейна-Подольского-Розена, образованных мировыми линиями зацепленных пар частиц $\{1,2\}$ и $\{3,4\}$. 
В работе [4] была предложена простая кинетическая модель реакции рождения и рекомбинации спиновых радикальных пар, учитывающая перебросы спиновых корреляций. При этом рассматривался элементарный акт, более общий, чем представленный на рис. 1. Для описания кинетической модели [4] введем так называемые состояния Вернера [5] для пары $\{i, j\}$ частиц со спином $1 / 2$ :

$$
\hat{\varrho}^{(x)}(i, j)=\frac{1-x}{4} \hat{I}(i, j)+x \widehat{P}_{0}(i, j) .
$$

Здесь $\hat{I}(i, j)$ - единичный оператор в объединенном спиновом пространстве частиц $i$ и $j$, $\widehat{P}_{0}(i, j)$ - проектор на синглетное состояние пары $\{i, j\}$, параметр $x \in[-1 / 3,1]$. Значение $x=1$ отвечает чистому синглетному состоянию, $x=0$ соответствует максимально смешанному спиновому состоянию пары $\{i, j\}$, а при $x=-1 / 3$ матрица плотности (1.3) описывает изотропное триплетное состояние. Разным типам спиновых корреляций в паpe $\{i, j\}$ отвечают различные значения параметра $x$ : при $x \in(1 / 3,1]$ состояние (1.3) оказывается зацепленным, а при $x \in[-1 / 3,1 / 3]$ (за исключением точки $x=0$ ) в нем присутствуют только классические корреляции типа (1.2) [6].

Для описания процесса, происходящего при перекрестной рекомбинации (по синглетному каналу) частиц из пар $\{1,2\}$ и $\{3,4\}$, удобно ввести отображение

$$
\hat{\varrho}^{(\text {pre })}(1,2,3,4) \mapsto \hat{\varrho}^{(\text {post })}(2,4)
$$

из множества изначальных спиновых состояний четверки частиш $\{1,2,3,4\}$ в множество состояний пары $\{2,4\}$, оставшейся после рекомбинации частиц 1 и 3 . Акт рекомбинации пары $\{1,3\}$ тождествен нулевому исходу при измерении спина этих частиц. Поэтому в соответствии с проекционным постулатом фон Неймана

$$
\hat{\varrho}^{\text {(post) }}(2,4)=\frac{\operatorname{Tr}_{1,3}\left[\widehat{P}_{0}(1,3) \hat{\varrho}^{(\text {pre })}(1,2,3,4)\right]}{\operatorname{Tr}_{1,2,3,4}\left[\widehat{P}_{0}(1,3) \varrho^{(\text {pre })}(1,2,3,4)\right]} .
$$

В работе [4] показано, что на подмножестве прямых произведений состояний Вернера (1.3) отображение (1.4) мультипликативно по параметру $x$ :

$$
\hat{\varrho}^{(x)}(1,2) \otimes \hat{\varrho}^{(y)}(3,4) \mapsto \hat{\varrho}^{(x y)}(2,4),
$$

что отражено на рис. 2

В модели [4] радикалы рождаются, диффундируют и рекомбинируют при дополнительном предположении, что акты диссоциации поставляют в среду только пары, находящиеся в синглетном или изотропном триплетном состояниях. В настоящей работе осуществлено обобщение модели [4] - допускается рождение пар в любых состояниях Вернера.

Существует один важный аспект проблемы описания переброса спиновых корреляций при рекомбинации радикалов, не получивший освешения в работе [4], а именно: что 


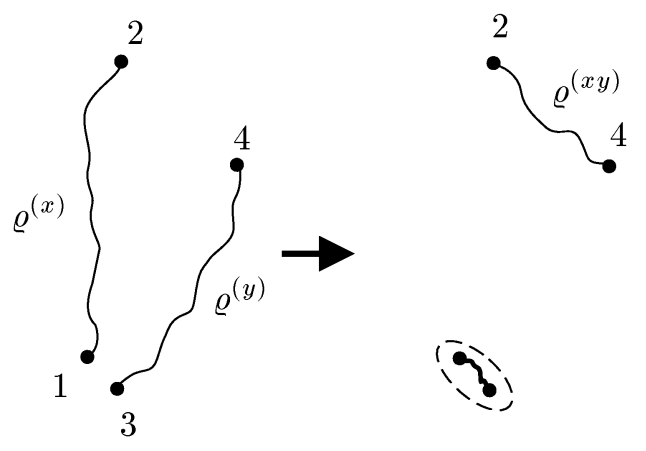

Рис. 2. Переброс спиновых корреляций при перекрестной рекомбинации частиц из двух пар, находящихся в состояниях Вернера. Скоррелированные пары соединены волнистыми линиями. Штриховой линией окружен продукт рекомбинации.

происходит со спиновыми состояниями пар $\{1,2\}$ и $\{3,4\}$ при встрече частиц 1 и 3 , не завершившейся их рекомбинацией? Не следует ли в этом случае считать, что пара $\{1,3\}$ оказывается в триплетном (изотропном) состоянии? Если ответить на этот вопрос положительно, кинетическая модель резко усложнится. Кроме скоррелированных пар в среде появляются скоррелированные четверки, шестерки, $\ldots, 2 n$-ки частиц. Противоположная точка зрения постулирует, что сближение частиц не приводит их автоматически в положение, из которого с вероятностью 0.25 (вероятность “синглетной” ситуации) происходит рекомбинация, т.е. предполагается, что существуют дополнительные не контролируемые внешним окружением факторы, обусловливающие рекомбинацию. В соответствии с этим при не завершившейся рекомбинацией встрече a priori нескоррелированных частищ в окружающий мир поступает исчезающе малый объем информации об их спиновом состоянии и частицы после расхождения остаются практически нескоррелированными. Необходимым условием правомерности такого взгляда является крайне малая вероятность рекомбинации при встрече радикалов. В настоящей работе принята именно такая точка зрения. В последнем разделе, однако, кратко обсуждены перспективы более строгого подхода к отмеченной проблеме.

\section{2. КИНЕТИЧЕСКАЯ МОДЕЛЬ С ОБОБШЕННЫМ ИСТОЧНИКОМ РАДИКАЛЬНЫХ ПАР}

Перейдем к описанию кинетической модели. В основных чертах она повторяет модель из работы [4]. Рассмотрим безграничную среду, в которой происходят акты рождения и рекомбинации пар частиц со спином $1 / 2$. В промежутках между актами рождения и рекомбинации частицы диффундируют с коэффициентом диффузии $D$, который для простоты считается одинаковым для обоих фрагментов рождаюшихся пар. Введем в рассмотрение набор функций $f^{(x)}\left(\mathbf{r}_{1}, \mathbf{r}_{2}\right)$. Это одновременно двухкоординатные пространственные плотности пар частиц и распределения по параметру $x$ пар, находяшихся 
в спиновом состоянии $\varrho^{(x)}(1.3)$. Напомним, что в выбранной модели спиновые коррелящии сушествуют только внутри некоторых пар частищ, но не в бо́льших кластерах. В рамках простейшего кинетического закона типа “действуюших масс" плотности пар подчиняются следуюшему кинетическому уравнению:

$$
\begin{aligned}
\partial_{t} f^{(x)}\left(\mathbf{r}_{1}, \mathbf{r}_{2}\right)= & -\nu f\left(\mathbf{r}_{1}\right) f^{(x)}\left(\mathbf{r}_{1}, \mathbf{r}_{2}\right)-\nu f^{(x)}\left(\mathbf{r}_{1}, \mathbf{r}_{2}\right) f\left(\mathbf{r}_{2}\right)+ \\
& +\nu \int_{x^{\prime} y^{\prime}=x} d x^{\prime} d y^{\prime} \int d^{3} r^{\prime} f^{\left(x^{\prime}\right)}\left(\mathbf{r}_{1}, \mathbf{r}^{\prime}\right) f^{\left(y^{\prime}\right)}\left(\mathbf{r}^{\prime}, \mathbf{r}_{2}\right)+ \\
& +D\left(\Delta_{\mathbf{r}_{1}}+\Delta_{\mathbf{r}_{2}}\right) f^{(x)}\left(\mathbf{r}_{1}, \mathbf{r}_{2}\right)+\gamma^{(x)}\left(\mathbf{r}_{1}\right) \delta\left(\mathbf{r}_{1}-\mathbf{r}_{2}\right)
\end{aligned}
$$

Здесь первые два члена в правой части отвечают за гибель пары при рекомбинации одной из ее частиц, находящихся в точках $\mathbf{r}_{1}$ и $\mathbf{r}_{2}$,

$$
f(\mathbf{r})=\int_{-\frac{1}{3}}^{1} d x \int d^{3} r^{\prime} f^{(x)}\left(\mathbf{r}, \mathbf{r}^{\prime}\right)
$$

- полная плотность рекомбинируюших частиц, константа $\nu$ задает частоту рекомбинаций, третий член отвечает за рождение новой скоррелированной пары при гибели двух других пар. Конкретная структура интеграла по параметрам состояний Вернера описана ниже. Параметры рожденной пары связаны с параметрами погибших отображением (1.6). Последние два слагаемых - диффузионные члены и источник локального рождения пар с неконкретизированной пока зависимостью от $x$. Рассматриваем пространственно однородную и стационарную ситуацию, при которой $f^{(x)}\left(\mathbf{r}_{1}, \mathbf{r}_{2}\right)=$ $f^{(x)}\left(\left|\mathbf{r}_{1}-\mathbf{r}_{2}\right|\right), f(\mathbf{r})=f, \gamma^{(x)}(\mathbf{r})=\gamma^{(x)}$. Для $f_{q}^{(x)}$ - пространственного фурье-образа функции $f^{(x)}\left(\left|\mathbf{r}_{1}-\mathbf{r}_{2}\right|\right)$ - возникают нелинейные интегральные уравнения, которые в зависимости от величины параметра $x$ приобретают, как нетрудно проверить, одну из следующих трех форм:

при $1 / 9 \leqslant x \leqslant 1$ :

$$
f_{q}^{(x)}=\alpha_{q}^{(x)}+\beta_{q} \int_{x}^{1} \frac{d y}{y} f_{q}^{(x / y)} f_{q}^{(y)}
$$

при $0 \leqslant x<1 / 9$ :

$$
f_{q}^{(x)}=\alpha_{q}^{(x)}+\beta_{q} \int_{x}^{1} \frac{d y}{y} f_{q}^{(x / y)} f_{q}^{(y)}-\beta_{q} \int_{-\frac{1}{3}}^{-3 x} \frac{d y}{y} f_{q}^{(x / y)} f_{q}^{(y)}
$$

при $-1 / 3 \leqslant x<0$ :

$$
f_{q}^{(x)}=\alpha_{q}^{(x)}+\beta_{q} \int_{-3 x}^{1} \frac{d y}{y} f_{q}^{(x / y)} f_{q}^{(y)}-\beta_{q} \int_{-\frac{1}{3}}^{x} \frac{d y}{y} f_{q}^{(x / y)} f_{q}^{(y)} .
$$

Здесь

$$
\alpha_{q}^{(x)}=\frac{\gamma^{(x)}}{2 \nu f+2 D q^{2}}, \quad \beta_{q}=\frac{\nu}{2 \nu f+2 D q^{2}}, \quad f=\sqrt{\int_{-\frac{1}{3}}^{1} d x \frac{\gamma^{(x)}}{\nu}}
$$




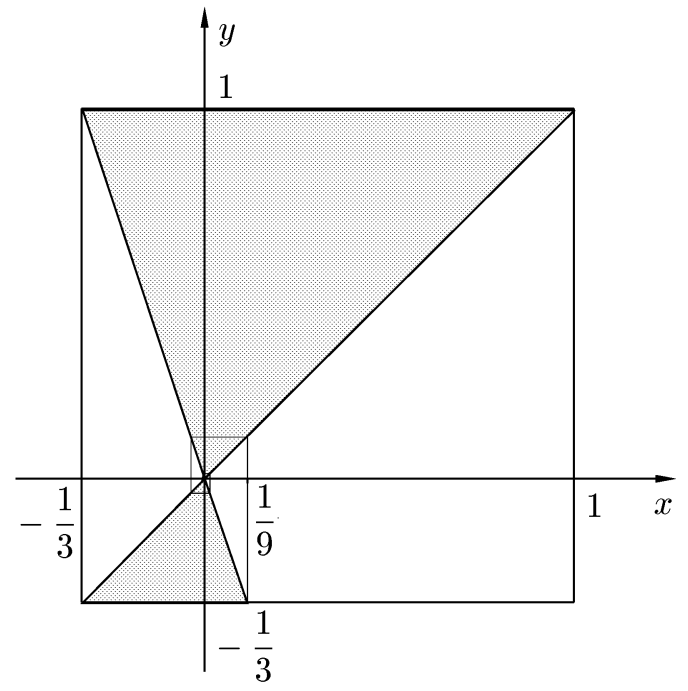

Рис. 3. Область интегрирования по $y$ в уравнениях (2.2)-(2.4) в зависимости от $x$ заштрихована.

Зависимость от $x$ пределов интегрирования проиллюстрирована на рис. 3 . Расстановка знаков перед интегралами в (2.2)-(2.4) диктуется необходимой положительностью членов, описываюших рождение пар.

Нелинейность представленных уравнений существенна только в (2.2). Действительно, пусть известно решение $f_{q, 1}^{(x)}$ этого уравнения на отрезке $\Delta_{1}=[1 / 9,1]$ оси $x$. Тогда для решения $f_{q, 2}^{(x)}$ на отрезке $\Delta_{2}=[-1 / 3,1 / 27]$ имеет место линейное уравнение

$$
f_{q, 2}^{(x)}=\alpha_{q}^{(x)}-2 \beta_{q} \int_{-\frac{1}{3}}^{x} \frac{d y}{y} f_{q, 1}^{(x / y)} f_{q, 2}^{(y)}
$$

На следуюшем шаге на отрезке $\Delta_{3}=[1 / 81,1 / 9]$ имеем

$$
f_{q, 3}^{(x)}=\alpha_{q}^{(x)}-\beta_{q} \int_{-\frac{1}{3}}^{-3 x} \frac{d y}{y} f_{q, 2}^{(x / y)} f_{q, 2}^{(y)}+\beta_{q} \int_{x}^{\frac{1}{9}} \frac{d y}{y} f_{q, 2}^{(x / y)} f_{q, 3}^{(y)}+\beta_{q} \int_{\frac{1}{9}}^{1} \frac{d y}{y} f_{q, 3}^{(x / y)} f_{q, 2}^{(y)}
$$

И так далее, на каждом отрезке $\Delta_{2 n}=\left[(-1 / 3)^{2 n-1},(-1 / 3)^{2 n+1}\right]$ и $\Delta_{2 n+1}=$ $\left[(-1 / 3)^{2 n+2},(-1 / 3)^{2 n}\right]$ решение определяется линейным интегральным уравнением с учетом всех предыдущих решений. Частное решение, приведенное в [4], соответствует фурье-образу $f_{q}^{(x)}$, сосредоточенному в концевых точках указанного множества отрезков. Эти конщевые точки образуются при пересечении оси $x$ с ломаной спиралью, часть которой изображена на рис. 3 .

Рассмотрим подробнее первый шаг - решение уравнения (2.2). Его удобнее рассматривать на всем отрезке $[0,1]$. Заметим, что оно дает также окончательное решение для 
ситуации, когда $\gamma^{(x)}=0$ при $x<0$. Введя новую переменную $\xi=-\ln x$ и переходя к фурье-образам

$$
f_{q}(\eta)=\int_{0}^{\infty} f_{q}^{(\exp (-\xi))} \exp (-i \xi \eta) d \xi, \quad \alpha_{q}(\eta)=\int_{0}^{\infty} \alpha_{q}^{(\exp (-\xi))} \exp (-i \xi \eta) d \xi
$$

получаем из (2.2) простое уравнение

$$
f_{q}(\eta)=\alpha_{q}(\eta)+\beta_{q} f_{q}^{2}(\eta)
$$

Его устойчивое решение

$$
f_{q}(\eta)=\frac{1}{2 \beta_{q}}\left(1-\sqrt{1-4 \alpha_{q}(\eta) \beta_{q}}\right)
$$

позволяет выразить $f_{q}^{(x)}$ через $n$-кратные свертки $\left[\alpha_{q}\right]_{n}^{(x)}$ функции $\alpha_{q}^{(x)}$ по переменной $-\ln x$ :

$$
f_{q}^{(x)}=\alpha_{q}^{(x)}+2 \sum_{n=1}^{\infty} \frac{(2 n-1) !}{(n+1) !(n-1) !} \beta_{q}^{n}\left[\alpha_{q}\right]_{n}^{(x)} .
$$

Используя это решение на отрезке $\Delta_{1}$, можно продвигаться дальше (на отрезки $\Delta_{2}$, $\left.\Delta_{3}, \ldots\right)$, решая уже только линейные уравнения.

\section{3. ОБСУЖДЕНИЕ}

Предложенная в [4] и обобшенная в настояшей работе кинетическая модель переброса квантовых спиновых корреляций относительно проста, что достигнуто ценой ряда принципиальных постулатов. Отметим их. Прежде всего, модель не учитьвает утраты спиновых корреляций пар в промежутках между актами рождения и рекомбинации. Эти утраты неизбежны в результате межчастичных столкновений и прецессии спинов во внешнем неоднородном магнитном поле. Члены, отвечаюшие за такого рода эффекты, нетрудно ввести в уравнение (2.1), что, естественно, усложнит модель, но не изменит ее радикально.

Более принципиальным моментом является предположение о классичности движения частиц. При этом считается, что положение частиц контролируется с точностью, достаточной для безошибочной идентификации пар, которым принадлежат прорекомбинировавшие радикалы. Неизбежные квантовые ограничения на точность локализации акта рекомбинации потребуют перераспределения переброшенных корреляший (с некоторыми весами) между несколькими новыми парами, которые все вместе будут образовывать скоррелированньй по спину кластер.

Эта проблема тесно примыкает к отмеченной в разделе 1 проблеме влияния несвершившихся рекомбинаций на информацию о спиновых состояниях частиц, поступаюшую в окружаюший мир. В строгом подходе пребывание в течение конечного времени пары 
$\{i, j\}$ в положении, из которого возможна рекомбинация, должно автоматически приводить к переоценке в сторону увеличения веса триплетности в их совместном спиновом состоянии:

$$
\hat{\varrho}^{(x)}(i, j) \mapsto \hat{\varrho}^{(y)}(i, j),
$$

где $x>y$. При этом, как уже отмечалось, неизбежно меняются спиновые корреляции меж ду всеми частицами, с которыми были скоррелированы радикалы $i$ и $j$. Такого рода информационные процессы в настояшей модели не могут быть учтены. Для их описания необходимо с самого начала оперировать с матрицей плотности всех радикалов, число которых не фиксировано, т.е. нужно строить вторично квантованную полевую модель хотя бы в ее простейшем дискретном варианте, который включает в себя рождения и рекомбинации синглетных пар, происходящие внутри ячеек, и миграцию отдельных радикалов между ячейками ${ }^{2)}$. Такая модель может учитьвать как квантовый характер миграции частиц, так и пространственную размытость акта рекомбинации в силу невозможности уточнения положения частиц внутри ячеек.

Среди образуюших алгебры наблюдаемых вторично квантованной модели должны присутствовать операторы рождения $\widehat{\Psi}_{i, j}^{\dagger}$ и уничтожения $\widehat{\Psi}_{i, j}$ синглетной пары, один фрагмент которой находится в ячейке $i$, а второй - в ячейке $j$. Аналогом двухкоординатной плотности синглетных пар служит величина

$$
\left\langle\widehat{\Psi}_{i, j}^{\dagger} \widehat{\Psi}_{i, j}\right\rangle_{\hat{\rho}} \equiv \operatorname{Tr}\left(\widehat{\Psi}_{i, j} \hat{\rho} \hat{\Psi}_{i, j}^{\dagger}\right)
$$

где $\hat{\rho}$ - полная матрица плотности многочастичной системы. Выраженный на языке операторных средних (3.2) процесс, изображенный на рис. 1, приводит к некоторому простому универсальному неравенству. Выведем его. Вместо четверки частиц будем рассматривать ячейки $1,2,3,4$. Если между частицами, находящимися в ячейках 1 и 2 (также как и между частицами из ячеек 3 и 4), сушествуют спиновые корреляции, возникшие из-за миграции фрагментов синглетных пар, то факт рекомбинации синглетной пары из ячеек 1,3 должен изменить априорную вероятность рекомбинации пары из ячеек 2,4 . Пусть $\hat{\rho}$ - начальная матрица плотности системы. После первого акта рекомбинации матрица плотности претерпит преобразование

$$
\hat{\rho} \mapsto \frac{\widehat{\Psi}_{1,3} \hat{\rho} \widehat{\Psi}_{1,3}^{\dagger}}{\left\langle\widehat{\Psi}_{1,3}^{\dagger} \widehat{\Psi}_{1,3}\right\rangle_{\hat{\rho}}} .
$$

Вероятность найти синглетную пару с фрагментами в ячейках 4,2 , задаваемая средним значением оператора $\widehat{\Psi}_{4,2}^{\dagger} \widehat{\Psi}_{4,2}$, оказывается больше для правой части отображения (3.3). Следовательно,

$$
\left\langle\widehat{\Psi}_{1,3}^{\dagger} \widehat{\Psi}_{1,3} \widehat{\Psi}_{4,2}^{\dagger} \widehat{\Psi}_{4,2}\right\rangle_{\hat{\rho}} \geqslant\left\langle\widehat{\Psi}_{1,3}^{\dagger} \widehat{\Psi}_{1,3}\right\rangle_{\hat{\rho}}\left\langle\widehat{\Psi}_{4,2}^{\dagger} \widehat{\Psi}_{4,2}\right\rangle_{\hat{\rho}} .
$$

\footnotetext{
2) Ячеечная модель ближе к реальной ситуации, чем может показаться с первого взгляда: она отражает существенные черты геминальной (клеточной) рекомбинации радикалов в растворах.
} 
Хотя акты рекомбинации локализованы в ячейках (т.е. следует отождествить ячейки 1 и 3), это неравенство записано в форме, явно симметричной относительно перестановки пар ячеек $\{1,3\}$ и $\{4,2\}$.

Получение строгих результатов в рамках вторично квантованной модели остается предметом дальнейших исследований.

Благодарности. Автор признателен П. Л. Чаповскому за стимулируюшее обсуждение затронутых в статье проблем. Работа выполнена при частичной финансовой поддержке Российского фонда фундаментальных исследований (грант № 98-02-17924), Федеральной целевой программы "Интеграция" (проект № 274) и программы "Университеты России" (проект № 015-01-01-30).

\section{Список литературы}

[1] S. Bell. Physics. 1964. V. 1. P. 195; J. Clauser, M. Horne, A. Shimony, R. Holt. Phys. Rev. Lett. 1969. V. 23. P. 880.

[2] M. Zukowski, A. Zeilinger, M. A. Horne, A. K. Ekert. Phys. Rev. Lett. 1993. V. 71. P. 4287.

[3] B. Brocklehurst. Int. Rev. Phys. Chem. 1985. V. 4. P. 279.

[4] Л. В. Ильичёв. ЖЭТФ. 2000. Т. 117. № 1. С. 248.

[5] R. F. Werner. Phys. Rev. A. 1989. V. 40. P. 4277.

[6] C.-H. Bennett, D.P. DiVincenzo, J. Smolin, W.K. Wootters. Phys. Rev. A. 1996. V. 54. P. 3824 . 